\title{
Spectroscopy and serial diffusion MR findings in hGH- Creutzfeldt-Jakob disease
}

\author{
C Oppenheim, M Zuber, D Galanaud, M Detilleux, F Bolgert, J L Mas, J Chiras, J F Meder
}

J Neurol Neurosurg Psychiatry 2004;75:1066-1069. doi: 10.1136/jnnp.2003.020172

This report describes the magnetic resonance imaging (MRI) findings in two patients fulfilling the diagnostic criteria for human growth hormone Creutzfeldt-Jakob disease, who initially had pronounced bilateral DWI/FLAIR (diffusion weighted imaging/fluid attenuated inversion recovery) hyperintensities in the basal ganglia, with decreased apparent diffusion coefficient (ADC) values (range, 58-82\% of normal). MRI spectroscopy, obtained in one case, showed decreased $\mathrm{N}$-acetyl aspartate/creatine (NAA/Cr) ratio in the atrophic vermis $(0.79$; normal: mean, 1.20; SD, 0.13), despite the lack of DWI/FLAIR signal changes, whereas NAA/Cr was normal in the putamina (1.6; normal: mean, 1.56; SD, 0.17), despite striking DWI signal changes and decreased ADC values (60\% of normal). Serial DWI, obtained in the other case, showed a progressive disappearance of DWI hypersignal of the basal ganglia replaced by pronounced atrophy. Data from these two patients suggest that restricted diffusion associated with a normal NAA value might indicate spongiform changes of still viable cells, and that any subsequent regression of the DWI signal changes, atrophy, or decreased NAA values could be related to progressive neuronal death.

C eutzfeldt-Jakob disease (CJD) is difficult to diagnose and image by conventional methods. In the past few years, diffusion weighted imaging (DWI) has been proposed as a useful tool for the early diagnosis of both sporadic and new variant forms of CJD, by demonstrating signal changes in the cortex and the basal ganglia. ${ }^{1-6}$ Recently, these changes were also seen in two patients with probable iatrogenic CJD, following corneal transplant ${ }^{7}$ and human growth hormone treatment. ${ }^{8}$ Magnetic resonance imaging (MRI) spectroscopy is a non-invasive method of probing brain metabolites in vivo. Although brain metabolite disorders have already been reported in CJD using MRI spectroscopy, ${ }^{9-11}$ their importance and extent are still speculative. We report two patients who met the World Health Organisation criteria for iatrogenic $\mathrm{CJD}^{12}$ and who were imaged with DWI and spectroscopy in one case and serial DWI in the other case, and discuss the imaging results in light of the known iatrogenic CJD data.

\section{CASE 1}

A 31 year old man initially developed gait unsteadiness, diplopia, and asthenia, which gradually worsened over a period of six months. Because of concentration difficulties the patient was unable to carry on working. On admission, he was slightly dysarthric, with a pronounced cerebellar syndrome including dizziness and ataxic gait. Ophthalmoplegia was not present and there were no pyramidal or extrapyramidal signs. The Mini Mental State examination was 29 of 30. The patient's medical history was unremarkable, except for serial injections of human growth hormone (hGH) between the ages of 15 and 17 years (from 1985 to 1987) for short stature. The electroencephalogram (EEG) was normal without periodic sharp wave complexes. The cerebrospinal fluid (CSF) was normal except for the detection of 14-3-3 protein by western blot analysis. Genotyping of the human prion protein (PRNP) gene revealed methionine/ valine heterozygosity at codon 129 .

Brain magnetic resonance imaging (MRI) was performed on a 1.5-T MRI unit (GE Signa Horizon Echospeed), including $\mathrm{T} 1$ and $\mathrm{T} 2$ weighted pulse sequences, a fluid attenuated inversion recovery (FLAIR) sequence, a DWI sequence $\left(b=1000 \mathrm{~s} / \mathrm{mm}^{2}\right)$, and a four single voxel spin echo MRI spectroscopic acquisition (PRESS; TR = $1500 \mathrm{ms,}$ $\mathrm{TE}=135 \mathrm{~ms}$ ). For spectroscopy, voxels, ranging from 6 to $8 \mathrm{~cm}^{3}$, were positioned in the thalami, the right lenticular nucleus, the parieto-occipital grey matter, and the vermis. Bilateral and symmetrical hyperintensities involving the caudate nuclei and the putamen were seen on FLAIR and T2 weighted images, without signal changes on T1 weighted images. Hyperintensities in the putamen and the caudate nuclei were more obvious on DWI, which also revealed bilateral hyperintensities in the thalami. The apparent diffusion coefficient (ADC) was very much decreased in the caudate nuclei and the putamen, and moderately decreased in the thalami (table 1; fig 1). The vermis was morphologically atrophic, with no signal changes on FLAIR or DWI, although ADC values in the vermis were slightly increased. As detailed in table 1, MRI spectroscopy showed a decreased $\mathrm{N}$-acetylaspartate/creatine $(\mathrm{NAA} / \mathrm{Cr})$ ratio in the vermis. Conversely, MRI spectroscopy failed to detect decreases in $\mathrm{NAA} / \mathrm{Cr}$ ratio in the areas with striking signal changes-that is, the putamina. The NAA/Cr ratio was at the lowest limit of normal values in the thalami. Choline/Cr ratios were within the range of normal values in the different areas.

\section{CASE 2}

A 31 year old man was admitted to hospital with an eight month history of progressive asthenia associated with major cerebellar signs, myoclonia, and memory impairment. His medical history was unremarkable except for serial injections of hGH between the ages of 14 and 15 years (from 1983 to 1984) for short stature. EEG showed diffuse aperiodic slow waves. The CSF was normal except for the detection of 14-3-3 protein. Genotyping of the PRNP gene revealed methionine/ valine heterozygosity at codon 129 .

\footnotetext{
Abbreviations: $A D C$, apparent diffusion coefficient; CJD, CreutzfeldtJakob disease; $\mathrm{Cr}$, creatine; CSF, cerebrospinal fluid; DWI, diffusion weighted imaging; EEG, electroencephalogram; FLAIR, fluid attenuated inversion recovery; $h G H$, human growth hormone; MRI, magnetic resonance imaging; NAA, N-acetyl aspartate; PRNP, human prion protein gene
} 

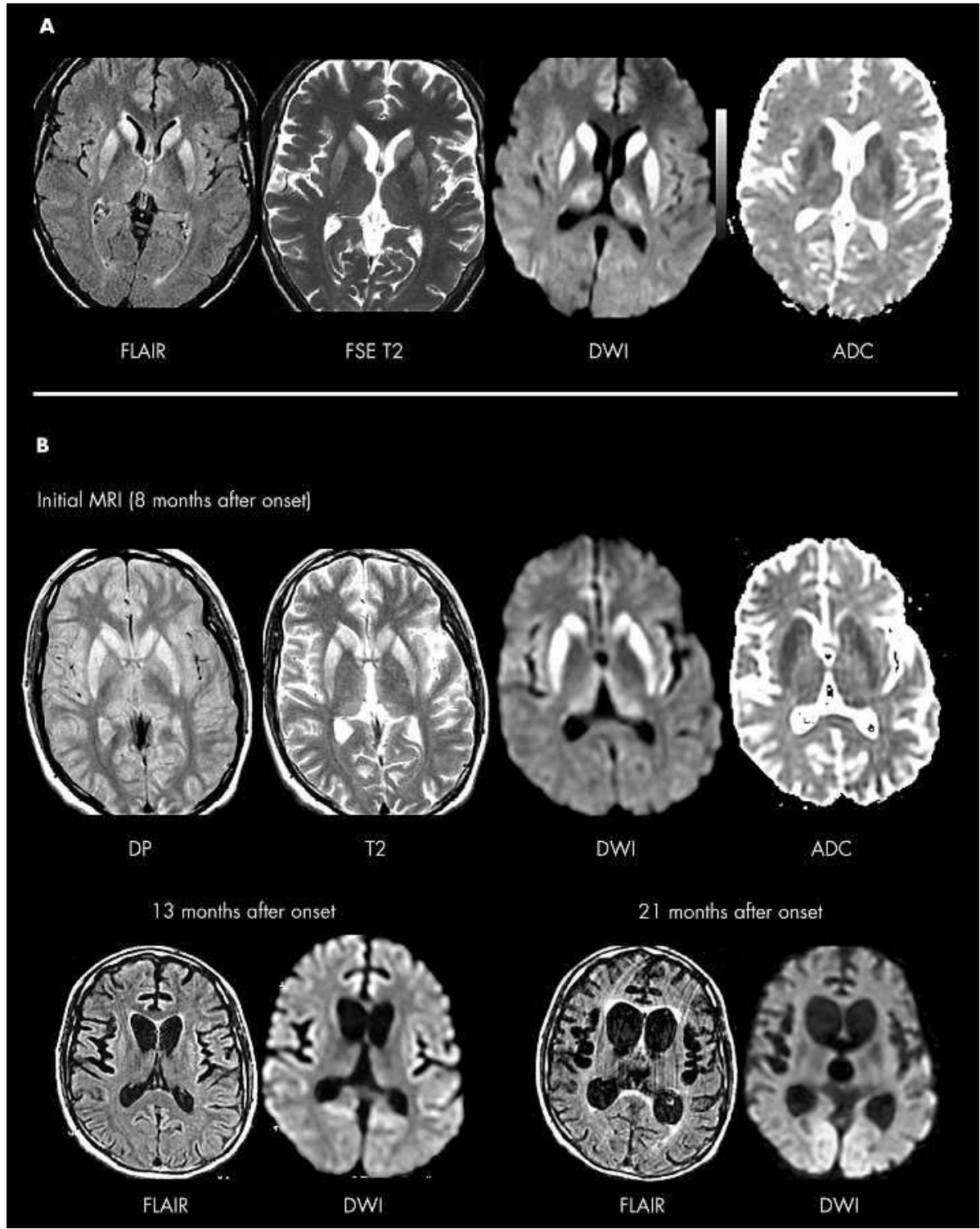

Figure 1 Magnetic resonance imaging (MRI) findings in two cases of iatrogenic Creutzfeldt-Jakob disease. (A) In case 1, fluid attenuated inversion recovery (FLAIR) and T2 weighted pulse sequences revealed bilateral and symmetrical hyperintensities in the caudate nucleus and putamen. These signal changes were more obvious on diffusion weighted images (DWI), which also revealed bilateral hyperintensities in the thalami. The apparent diffusion coefficient $(A D C)$ was very much decreased in the caudate nucleus and putamen, and moderately decreased in the thalami. (B) In case 2 , eight months after onset, a pronounced signal increase on T2 weighted, FLAIR, and DWI sequences was seen bilaterally and symmetrically in the striatum and thalami, with decreased ADC values. On successive follow up MRIs (13 and 21 months after onset), the basal ganglia and the entire cerebellum (not illustrated) were extremely atrophic, with prominent ventricular enlargement and partial then complete regression of the signal changes initially observed on FLAIR and DWI MRI sequences.

On MRI, the vermis was slightly atrophic with no signal changes. A pronounced signal increase was seen bilaterally and symmetrically in the striatum, the thalami, and in the cortex of the frontal lobes on T2, FLAIR, and DWI (fig 1). The ADC was decreased in the striatum and the thalami (table 1). Thirteen months after onset, the patient was bedridden with progressive occurrence of tremor and mental deterioration. FLAIR and DWI hyperintensities of the basal ganglia and frontal cortex had resolved with shrinkage of the basal ganglia and enlargement of the ventricular system. Pronounced atrophy of the vermis, and to a lesser extent of the cerebellar hemispheres, was observed. On the 21 month follow up MRI, two months before death, the basal ganglia and the entire cerebellum were extremely atrophic, with prominent ventricular enlargement and complete regression of the signal changes initially seen on FLAIR and DWI MRI sequences.

\section{DISCUSSION}

Patients with iatrogenic CJD usually follow a different clinical course to sporadic CJD. Cerebellar manifestations are prominent, and the EEG may not present the classic periodic pattern. In $\mathrm{hGH}^{14}$ and dura-mater $\mathrm{CJD},{ }^{15}$ computed tomography and MRI are often unremarkable in the early stages of the disease, showing atrophy only in the final stages. However, the MRI protocol used in these patients did not include FLAIR and DWI sequences. ${ }^{14}{ }^{15}$ Using these MRI sequences, increased signal intensity bilaterally in the striatum was reported recently in two patients with probable CJD following corneal transplant ${ }^{7}$ and hGH treatment. $^{8}$ 
Table 1 Mean apparent diffusion coefficient (ADC), and $\mathrm{N}$-acetylaspartate/creatine (NAA/Cr) ratios from various regions of interest

\begin{tabular}{|c|c|c|c|c|c|}
\hline \multirow[b]{2}{*}{ Region } & \multicolumn{3}{|l|}{ Case 1} & \multicolumn{2}{|l|}{ Case 2} \\
\hline & $\begin{array}{l}\text { Signal } \\
\text { changes } \\
\text { on DWI }\end{array}$ & $\begin{array}{l}\mathrm{ADC}, 10^{6} \mathrm{~mm}^{2} / \mathrm{s}(\% \\
\text { of normal)* }\end{array}$ & NAA/Crt & $\begin{array}{l}\text { Signal changes } \\
\text { on initial DWI }\end{array}$ & $\begin{array}{l}\text { ADC, } 10^{6} \mathrm{~mm}^{2} / \mathrm{s} \\
(\% \text { of normal })^{*}\end{array}$ \\
\hline Caudate nucleus & +++ & $458(62.5 \%)$ & NA & +++ & $520(71 \%)$ \\
\hline Putamen & +++ & $419(60 \%)$ & $1.6(1.56 ; 0.17)$ & ++ & 407 (58\%) \\
\hline Thalamus & + & $565(76 \%)$ & $1.31(1.64 ; 0.35)$ & + & $605(82 \%)$ \\
\hline Vermis & - & 928 (124\%) & $0.79(1.20 ; 0.13)$ & - & $768(102 \%)$ \\
\hline Internal capsule & - & 705 (101\%) & NA & - & $687(98 \%)$ \\
\hline $\begin{array}{l}\text { Occipital grey } \\
\text { matter }\end{array}$ & - & $782(95 \%)$ & $1.91(1.88 ; 0.34)$ & - & 802 (97\%) \\
\hline \multicolumn{6}{|c|}{$\begin{array}{l}\text { *Mean quantitative ADC values were calculated on small }\left(30 \mathrm{~mm}^{2}\right) \text { circular regions of interest placed in the head of } \\
\text { the right caudate nucleus, and in the right thalamus, putamen, occipital lobe, and vermis. The MRI quantitative } \\
\text { diffusion data were compared with normal values obtained from } 5 \text { healthy, age matched volunteers using an } \\
\text { identical diffusion acquisition and post-processing method. }+M R I \text { spectroscopic data were compared with spectra } \\
\text { obtained from } 30 \text { healthy volunteers for the vermis }{ }^{13} \text { and those of } 5 \text { healthy volunteers for the supratentorial spectra } \\
\text { (data not shown); values in parenthesis are the mean and SD values for these controls. } \\
\text { DWI, diffusion weighted imaging; MRI, magnetic resonance imaging; NA, not available. }\end{array}$} \\
\hline
\end{tabular}

Similarly, we saw pronounced signal changes, most clearly visible with DWI, in the striatum in two iatrogenic CJD patients. In line with reported pathological data in hGH CJD, ${ }^{14}{ }^{16}{ }^{17}$ these two patients also presented a slight, bilateral, DWI hypersignal in the thalami, a previously unrecognised radiological pattern in hGH CJD. Thalamic signal changes have previously been reported in sporadic and new variant CJD. The subtle changes found here are similar to those seen in sporadic $\mathrm{CJD}^{18}$ but differ from the pronounced hyperintensities of the posterior thalami reported in new variant CJD. ${ }^{3}$

Serial DWI studies in CJD are of interest in trying to understand the physiopathology of MRI changes in CJD. The restricted diffusion (low ADC values) seen in CJD is thought to result from the presence of numerous vacuoles of variable size with spongiform cellular changes, which would hinder the molecular motion of water. ${ }^{1419}$ Accordingly, the low ADC values seen here in the striatum and the thalami of both patients at an early stage of the disease could indicate prominent spongiform changes, which would correspond to previously reported pathological data in hGH CJD. ${ }^{14}{ }^{16}$ The temporal changes in DWI signal described in sporadic CJD are conflicting. Progressive or constant signal changes can be seen, ${ }^{50}$ whereas, in line with the MRI data reported here, decreased signal changes ${ }^{2122}$ have been reported in about half of the patients, ${ }^{5}$ late in the course of the disease.

MRI spectroscopy studies have shown decreased NAA values in the brain of both animals ${ }^{9}$ and humans with sporadic $^{102324}$ or variant $^{24}{ }^{25} \mathrm{CJD}$. Decreased NAA, regarded as a marker of neuronal death, was described in the cortex, ${ }^{9}$ the basal ganglia, and the white matter ${ }^{23}$ - that is, the areas that most often appeared normal or atrophic on conventional MRI. Recently, decreased NAA was seen in frontal lobes, associated with a pronounced hypersignal on DWI, in a patient with a suspected iatrogenic form of CJD following corneal transplant, a few weeks before death. ${ }^{7}$ Interestingly, changes in metabolites detectable by proton MRI spectroscopy are not an early feature in CJD. ${ }^{7}{ }^{1023}$ We found normal $\mathrm{NAA} / \mathrm{Cr}$ ratios in regions that appeared bright on DWI-the putamina and thalami-suggesting the presence of vacuolised neurones without cell death. In contrast, a decrease in NAA was seen in the atrophic vermis with no DWI/FLAIR signal changes. These radiological data (atrophy and pronounced decrease in the NAA/Cr ratio) are compatible with extensive neuronal death. This highlights the capacity of MRI spectroscopy to reveal metabolic changes in symptomatic brain regions that lack signal changes.
Based on the combined data from the two cases reported here, we speculate that DWI hypersignal with low ADC values and normal $\mathrm{NAA} / \mathrm{Cr}$ ratios indicates prominent spongiform changes of still viable cells, and that any regression of the DWI signal changes, atrophy, and decreased NAA values that might subsequently occur are related to progressive neuronal death.

\section{ACKNOWLEDGEMENTS}

We thank Drs S Haik and JP Brandel for helpful discussions.

\section{Authors' affiliations}

C Oppenheim, J F Meder, Department of Neuroimaging, Sainte-Anne Hospital, 75014 Paris, France

M Zuber, J L Mas, Neurological Department, Sainte-Anne Hospital D Galanaud, J Chiras, Neuroradiological Department, Pitié-Salpêtrière Hospital, 75013 Paris, France

M Detilleux, Internal Medicine Department, Cochin Port-Royal Hospital, 75013 Paris, France

F Bolgert, Intensive Care Unit, Pitié-Salpêtrière Hospital

Conflicting interests: none declared

Correspondence to: $\operatorname{Dr} C$ Oppenheim, Département d'Imagerie Morphologique et Fonctionnelle, Centre Hospitalier Sainte-Anne, Université Paris V, 1 rue Cabanis, 75014 Paris, France; oppenheim@ chsa.broca.inserm.fr

Received 5 June 2003

In revised form 14 October 2003

Accepted 1 November 2003

\section{REFERENCES}

1 Demaerel P, Sciot R, Robberecht W, et al. Accuracy of diffusion-weighted MR imaging in the diagnosis of sporadic Creutzfeldt-Jakob disease. J Neurol 2003;250:222-5.

2 Matoba M, Tonami $\mathrm{H}$, Miyaji $\mathrm{H}$, et al. Creutzfeldt-Jakob disease: serial changes on diffusion-weighted MRI. J Comput Assist Tomogr 2001;25:274-7.

3 Zeidler M, Sellar R, Collie D, et al. The pulvinar sign on magnetic resonance imaging in variant Creutzfeldt-Jakob disease. Lancet 2000;355:1412-8.

4 Oppenheim C, Brandel J, Hauw J, et al. MRI and the second French case of vCJD. Lancet 2000;356:253-4.

5 Murata T, Shiga Y, Higano S, et al. Conspicuity and evolution of lesions in Creutzfeldt-Jakob disease at diffusion-weighted imaging. AJNR Am J Neuroradiol 2002;23:1164-72.

6 Bahn M, Parchi P. Abnormal diffusion-weighted magnetic resonance images in Creutzfeldt-Jakob disease. Arch Neurol 1999;56:577-83.

7 Rabinstein AA, Whiteman ML, Shebert RT. Abnormal diffusion-weighted magnetic resonance imaging in Creutzfeldt-Jakob disease following corneal transplantations. Arch Neurol 2002;59:637-9.

8 Caboclo L, Huang N, Lepski G, et al. latrogenic Creutzfeldt-Jakob disease following human growth hormone therapy: case report. Arq Neuropsiquiatr 2002;60:458-61. 
9 Behar K, Boucher R, Fritch W, et al. Changes in N-acetylaspartate and myoinositol detected in the cerebral cortex of hamsters with Creutzfeldt-Jakob disease. Magn Reson Imaging 1998;16:963-8.

10 Graham GD, Petroff OA, Blamire AM, et al. Proton magnetic resonance spectroscopy in Creutzfeldt-Jakob disease. Neurology 1993:43:2065-8.

11 Shyu W, Lee C, Hsu Y, et al. Panencephalitic Creutzfeldt-Jakob disease. Unusual presentation of magnetic resonance imaging and proton magnetic resonance spectroscopy. J Neurol Sci 1996;138:157-60.

12 WHO. Global surveillance, diagnosis and therapy of human transmissible spongiform encephalopathies: report of a WHO consultation. In: Emerging and other communicable diseases, surveillance and control. Geneva: World Health Organisation, 1998.

13 Galanaud D, Le Fur Y, Nicoli F, et al. Regional metabolite levels of the norma posterior fossa studied by proton chemical shift imaging. MAGMA 2001;13:127-33.

14 Billette de Villemeur T, Gelot A, Deslys JP, et al. latrogenic Creutzfeldt-Jakob disease in three growth hormone recipients: a neuropathological study. Neuropathol Appl Neurobiol 1994;20:111-17

15 Martinez-Lage J, Poza M, Sola J, et al. Accidental transmission of CreutzfeldtJakob disease by dural cadaveric grafts. J Neurol Neurosurg Psychiatry 1994;57:1091-4.

16 Weller RO, Steart PV, Powell-Jackson JD. Pathology of Creutzfeldt-Jakob disease associated with pituitary-derived human growth hormone administration. Neuropathol Appl Neurobiol 1986;12:117-29.
17 Tintner R, Brown P, Hedley-Whyte ET, et al. Neuropathologic verification of Creutzfeldt-Jakob disease in the exhumed American recipient of human pituitary growth hormone: epidemiologic and pathogenetic implications. Neurology 1986;36:932-6.

18 Schroter A, Zerr I, Henkel K, et al. Magnetic resonance imaging in the clinical diagnosis of Creutzfeldt-Jakob disease. Arch Neurol 2000;57:1751-7.

19 Bahn MM, Kido DK, Lin W, et al. Brain magnetic resonance diffusion abnormalities in Creutzfeldt-Jakob disease. Arch Neurol 1997;54:1411-15.

20 Uemura A, O'uchi T, Sakamoto T, et al. High signal of the striatum in sporadic Creutzfeldt-Jakob disease: sequential change on T2-weighted MRI. Neuroradiology 2002;44:314-18.

21 Matoba M, Tonami H, Miyaji H, et al. Creutzfeldt-Jakob disease: serial changes on diffusion-weighted MRI. J Comput Assist Tomogr 2001;25:274-7.

22 Hirose Y, Mokuno K, Abe Y, et al. A case of clinically diagnosed Creutzfeld Jakob disease with serial MRI diffusion weighted images. Rinsho Shinkeigaku 1998;38:779-82.

23 Bruhn $\mathrm{H}$, Weber T, Thorwirth V, et al. In-vivo monitoring of neuronal loss in Creutzfeldt-Jakob disease by proton magnetic resonance spectroscopy. Lancet 1991;29:1610-11.

24 Pandya HG, Coley SC, Wilkinson ID, et al. Magnetic resonance spectroscopic abnormalities in sporadic and variant Creutzfeldt-Jakob disease. Clin Radiol 2003;58:148-53.

25 Galanaud D, Dormont D, Grabli D, et al. MR spectroscopic pulvinar sign in a case of variant Creutzfeldt-Jakob disease. J Neuroradiol 2002;29:285-7. 